\title{
Distribución, tallas y proporción sexual del calamar Lolliguncula panamensis del Golfo de Tehuantepec, México
}

\section{Distribution, size and sexual proportion of squid Lolliguncula panamensis from Gulf of Tehuantepec, Mexico}

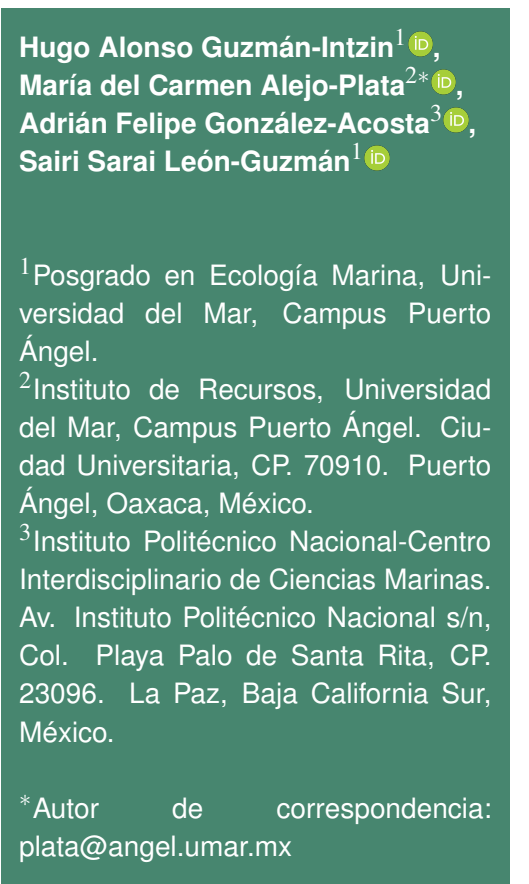

Artículo científico

Recibido: 29 de noviembre de 2019 Aceptado: 04 de febrero de 2020

Como citar: Guzmán-Intzin HA Alejo-Plata MC, González-Acosta AF, León-Guzmán SS (2020) Distribución, tallas y proporción sexual del calamar Lolliguncula panamensis del Golfo de Tehuantepec, México. Ecosistemas y Recursos Agropecuarios 7(1): e2484. DOI: 10.19136/era.a7n1.2484

RESUMEN. En el Golfo de Tehuantepec se desarrolla una intensa actividad pesquera centrada en la captura de camarones y peces demersales. Como parte de la pesca de arrastre de camarones, la captura de Lolliguncala panamensis es abundante. El objetivo fue evaluar la distribución, abundancia, estructura de tallas y proporción sexual del calamar dedal L. panamensis, en la zona de pesca del camarón en el Golfo de Tehuantepec. Los calamares se recolectaron de la fauna de acompañamiento del camarón, durante noviembre y diciembre de 2017 y febrero (temporada de pesca), mayo, julio y agosto de 2018 (temporada de veda); a partir de cinco viajes y 156 lances de pesca de altamar, realizados a bordo de embarcaciones camaroneras comerciales. Se recolectaron 746 hembras $(21$ a $110 \mathrm{~mm}$ Longitud del manto, LM) y 76 machos (21 a $93 \mathrm{~mm} \mathrm{LM}$ ), con la mayor abundancia (94.5\%), entre los 20 y $30 \mathrm{~m}$ de profundidad. Las hembras dominaron la proporción sexual $(1 \mathrm{M}: 7 \mathrm{H} \chi 2=248.81, \mathrm{p}<0.05)$, mientras que las diferencias en talla y peso entre ambos sexos, fueron significativas (K-S, $p<0.05)$. La abundante captura de esta especie en el Golfo de Tehuantepec, principalmente durante la veda del camarón (temporada de lluvias), sugiere la importancia de este calamar como un recurso potencial explotable y una alternativa para los pescadores durante la veda de otros recursos como el camarón y tiburón; así como una fuente de proteínas complementarias para las poblaciones locales.

Palabras clave: Chiapas, fauna de acompañamiento del camarón, pesquerías potenciales, Oaxaca.

ABSTRACT. An intense fishing activity focused to the capture of shrimp and demersal fishes is developed in the Gulf of Tehuantepec (Mexico). As part of the shrimp trawl fishing the capture of the Lolliguncula panamensis is very abundant. Assess the distribution, abundance, size structure and sexual proportion of the Panama brief squid, in the gulf of Tehuantepec, México. The squids specimens were collected through bycatch shrimp trawlers during November and December 2017, and February (fishing season), May, July and August 2018 (closed fishing season); from five fishing open ocean trips (156 fishing sets). A total of 746 females (21 to $110 \mathrm{~mm}$ Mantle length, LM) and 76 males (21 to $93 \mathrm{~mm} \mathrm{LM}$ ) were caught, with the highest abundance (94.5\%) between 20 and 30 m deep. Sexual proportion was predominated by females $(1 \mathrm{M}: 7 \mathrm{H} \chi 2=248.81, \mathrm{p}<0.05)$, whereas size-weight relationship differences were significant (K-S, $p<0.05)$. The abundant capture of L. panamensis in the Gulf of Tehuantepec, mainly during the fishing shrimp season (rainy season), suggests the importance of the Pacific brief squid as a potential exploitable resource as well as an alternative for fishermen during the fishing closure of resources like shrimp and sharks. Likewise, represents a complementary source of proteins for local populations.

Key words: By-catch shrimp fishery, Chiapas, potential fisheries, Oaxaca. 


\section{INTRODUCCIÓN}

El Golfo de Tehuantepec (GT) es una región de gran complejidad ecológica e intensa producción pesquera en el Pacífico oriental tropical [POT] (GallegosGarcía y Barberán-Falcón 1998). En el GT se desarrolla una intensa actividad pesquera, dirigida a la captura y aprovechamiento de recursos con alto valor económico, como el camarón y peces demersales (Tapia-García et al. 1994). En este contexto, las redes de arrastre utilizadas por la flota camaronera son eficientes para la captura de las tallas requeridas de camarón, lo que coadyuva al cuidado de las poblaciones de este recurso (DOF 2013). Pero debido a que la actividad de pesca se efectúa en áreas cercanas a la costa, donde se concentra una gran diversidad y abundancia de especies de peces e invertebrados, estos quedan atrapados incidentalmente en las redes de pesca (Tapia-García et al. 1994). Debido a que forman parte de la fauna de acompañamiento del camarón (FAC), la que por lo general no constituye el objeto principal de aprovechamiento de la pesquería (Alverson et al. 1994).

Como parte de la FAC, en el GT se capturan tres especies de calamares de la familia Loliginidae: Lolliguncula argus Brakoniecki y Roper, 1985, L. diomedeae Hoyle, 1904 y L. panamensis Berry, 1911. Cuando la captura de calamares es muy abundante, los ejemplares de mayor tamaño se comercializan para consumo en restaurantes locales, o son utilizados como carnada para la pesca de otras especies (Alejo-Plata et al. 2001). Por lo que estas especies de calamar pueden considerarse como un recurso con potencial pesquero para la región por su abundancia (Alejo-Plata et al. 2015).

El calamar dedal (L. panamensis) es una especie nerítica que habita en el POT, desde el Golfo de California en México hasta Perú, principalmente sobre la plataforma y talud continental, a profundidades entre 16 y 65 m (Sánchez 2003). De las especies de lolilginidos que habitan el POT, el calamar dedal es el más robusto y de mayor talla, por lo que es un recurso potencial y de interés pesquero (Alejo-Plata et al. 2001, Jereb et al. 2010). Debido a lo anterior esta especie ha sido objeto de estudios sobre su taxonomía (Cardozo y Hochberg 2013), hábitos de alimentación (Arizmendi-Rodríguez et al. 2011), reproducción (Arizmendi-Rodríguez et al. 2012a), así como abundancia y distribución (Arizmendi-Rodríguez et al. 2012b).

A pesar de su captura recurrente y abundancia como parte de la FAC en el GT, el conocimiento sobre los aspectos de la biología y dinámica poblacional del calamar dedal es poco. Por lo que el objetivo del presente estudio fue conocer la distribución, abundancia, estructura de tallas y proporción sexual de $L$. panamensis en la zona de pesca del camarón en el GT, México.

\section{MATERIALES Y MÉTODOS}

EI GT es un área tropical del sureste de México, se encuentra entre los $14^{\circ} 30^{\prime}-16^{\circ} 10^{\prime} \mathrm{LN}$ y $92^{\circ} 25^{\prime}-98^{\circ} 08^{\prime}$ LO; con longitud aproximada de $209 \mathrm{~km}$, y se caracteriza por la presencia de extensas barreras arenosas en su litoral. De mayo a junio se tienen tormentas tropicales y huracanes, que se extiende de octubre a noviembre (Reyes y MejíaTrejo 1991). Un fenómeno de gran importancia en el GT es el arribo entre noviembre y abril, de vientos del Norte o Tehuanos con intensidades mayores $10 \mathrm{~ms}^{-1}$ y duración típica de 2 a 6 días (Romero-Centeno et al. 2003). Estos episodios de viento contribuyen a que las aguas del golfo sean relativamente productivas y frías comparadas con sus alrededores (OrtegaGarcía et al. 2000).

El muestreo se realizó de noviembre a diciembre de 2017, y en febrero (temporada de pesca del camarón), mayo, julio y agosto de 2018 (temporada de veda del camarón). El área de estudio fue la zona de pesca 90 (Z90), situada entre las localidades de Punta Chipehua; Oaxaca ( $\left.16^{\circ} 10^{\prime} \mathrm{LN}-95^{\circ} 11^{\prime} \mathrm{LO}\right)$ y Puerto Madero, Chiapas ( $\left.14^{\circ} 41^{\prime} \mathrm{LN}-92^{\circ} 24^{\prime} \mathrm{LO}\right)$; la cual para la pesca del camarón se divide en cinco sub-zonas (S91-S95) (Figura 1) (INAPESCA 2012).

La recolección de calamar se realizó de la FAC obtenida de cinco viajes de pesca de altamar realizados en embarcaciones camaroneras comerciales, del programa Evaluación de las especies asociadas a la pesca de arrastre de camarón en el GT, México. Las 

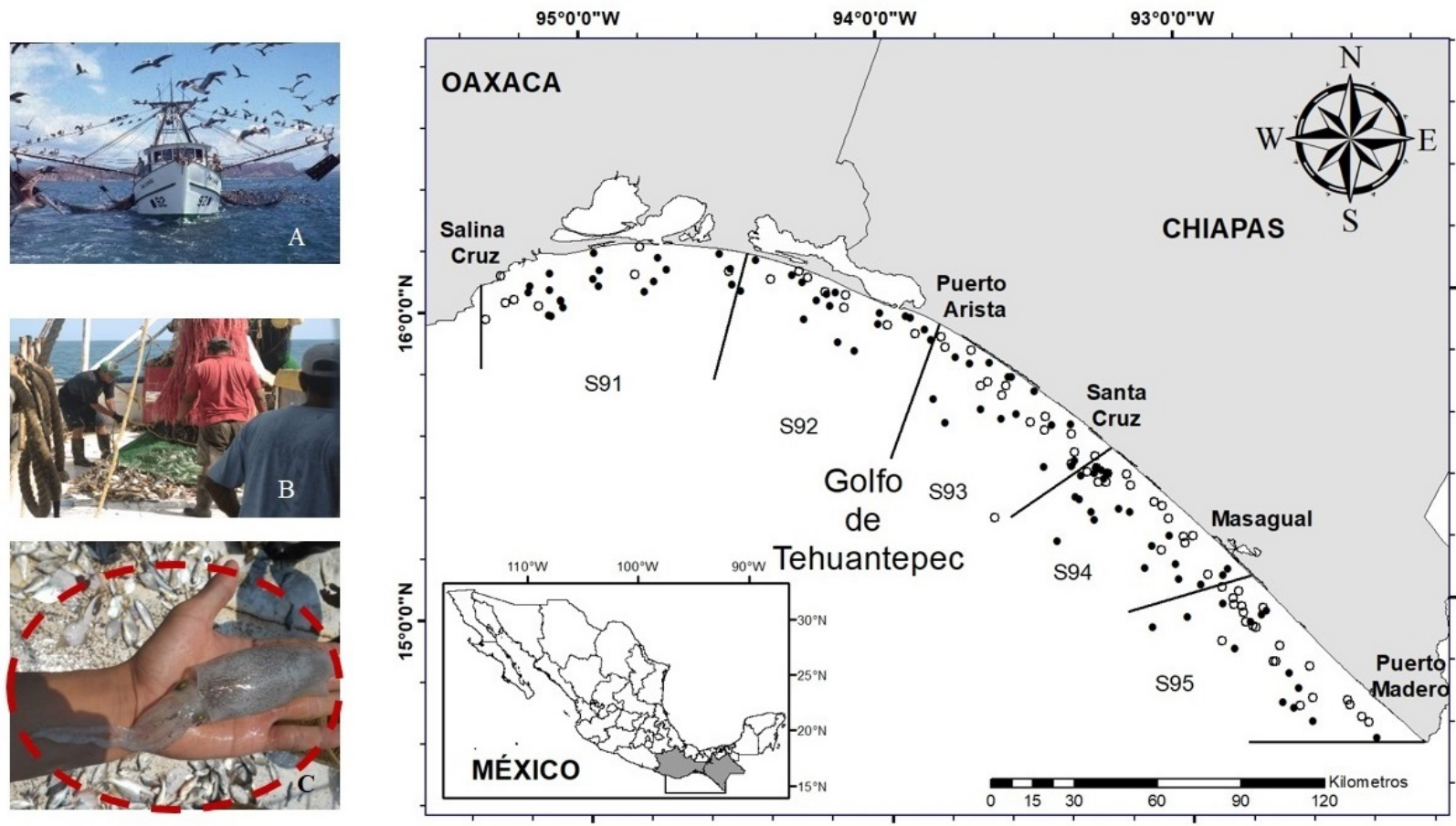

Figura 1. Área de estudio donde se indican las estaciones de muestreo (puntos negros) y las estaciones positivas para Lolliguncula panamensis (puntos blancos). A y B, maniobras de pesca, C L. panamensis

embarcaciones están equipadas con red de arrastre tipo camaronera de $27.4 \mathrm{~m}$ en relinga superior, luz de malla de $57.1 \mathrm{~mm}$ en el cuerpo y $33.9 \mathrm{~mm}$ en el copo, sostenida con tablas de madera de $25.4 \times 152.4 \mathrm{~cm}$; y excluidores. La recolecta se realizó en 156 estaciones, en sitios con profundidades entre 7 y $65 \mathrm{~m}$ (Figura 1); los arrastres tuvieron una duración de una hora a velocidad de 2 nudos. Cada lance de pesca se georefereferenció con un GPS Chart 421s Standard Horizon; mientras que la profundidad de captura se midió con un sonar FURUNO FCV-295. Siempre que fue posible, después de cada arrastre, la captura se vació en cubierta para obtener una muestra de FA de entre 30 y $40 \mathrm{~kg}$, de acuerdo a lo establecido por López-Martínez et al. (2010). La muestra se congeló a $-15{ }^{\circ} \mathrm{C}$ hasta su procesamiento en el Laboratorio de Oceanografía Biológica de la Universidad del Mar, donde los calamares se separaron de los peces, crustáceos, equinodermos y otros moluscos. La identificación taxonómica se realizó con base en los criterios de Jereb et al. (2010); a cada ejemplar de calamar se le midió la longitud dorsal del manto con un vernier digital $(\mathrm{LM}, \pm 0.1 \mathrm{~mm})$ y el peso total (PT, $\pm 0.1 \mathrm{~g}$ ) con una balanza digital OHAUS HH32O. De igual modo, cada organismo fue eviscerado para identificar el sexo y su estadio de madurez sexual como: inmaduros, madurando, maduros y desovados (Lipinski y Underhill 1995). En las hembras se determinó la presencia de ovocitos maduros en el ovario, mientras que en los machos se observó la presencia de espermatóforos. La cópula previa fue confirmada por la presencia de espermatangias en la cavidad del manto de las hembras.

\section{Análisis de datos}

Las longitudes de los calamares dedal capturados se representan en histogramas de frecuencias de longitud (histograma de tallas) con intervalos de 5 $\mathrm{mm}$; mientras que la descripción y análisis estadístico de las modas observadas, se realizó por medio de la función de densidad multinomial (Hastings y Peacock 1975). La optimización Solver se utilizó para ajustar los datos de tallas zonales (sub-zonas) y temporales (mes de muestreo) de cada intervalo a una, dos, tres, 
cuatro y cinco modas; de esta manera, la elección del número de modas por cada zona se realizó con la prueba Akaike (AIC).

Se utilizó el modelo $\mathrm{p}=\mathrm{aLM}^{b}$ para analizar los datos de longitud y peso $(a=$ pendiente y $b=$ intercepto), considerando a las hembras y machos por separado. Con el fin de aceptar (o rechazar) la hipótesis de crecimiento isométrico, se aplicó la prueba $t$ de Student para la comparación de la pendiente. La normalidad de los datos se determinó con las distribuciones de ambas variables, mediante una prueba Shapiro-Wilky; y las diferencias en longitud y peso, con la prueba Kolmogorov-Smirnov (Zar 1999).

La proporción de sexos se determinó con la relación hembras/machos $(\mathrm{H}: \mathrm{M})$ de la muestra analizada. Para determinar si la proporción de sexos se desviaba de la relación $1: 1$, se aplicó una $\chi^{2}$ cuadrada con la corrección de Yates (Zar 1999).

La información mensual para la TSM se obtuvo del catálogo de productos del sitio Copernicus Marine Environment Monitoring Service (Copernicus) 1 con nivel 4 de procesamiento del océano global y con alta resolución de $5 \mathrm{~km}$ por pixel. Por cada matriz de datos se realizaron pruebas de correlación múltiple, las variables obtenidas durante el estudio fueron dos en escala ordinal, mes y zona; una de intervalo: TSM; y de razón: abundancia, talla y peso. Debido a que las variables mes y zona son variables no métricas, se realizó la prueba estadística no paramétrica de Spearman con el programa Statistica ${ }^{M R}$ Versión 8.0 (Statsoft, Inc); y con la paquetería del programa de sistemas de información geográfica (SIG) Arcgis 10.2.1 se elaboraron mapas puntualizando la distribución de $L$. panamensis representando por tamaños la abundancia de los organismos.

\section{RESULTADOS}

De los 156 lances efectuados, el 45\% fueron positivos para la captura de L. panamensis (Figura 1). La mayor abundancia (94.5\%) se tuvo en la temporada de lluvias, contrastando con la mínima (5.5\%) en la temporada de secas (Tabla 1, Figura 2). Por lo que la abundancia varió de forma significativa $(p=$ 0.011 ) entre meses y periodos de muestreo.
En las variables ambientales, los promedios mensuales de la TSM fluctuaron entre $28{ }^{\circ} \mathrm{C}(\mathrm{DE}=$ $0.424)$ y $30.5^{\circ} \mathrm{C}(\mathrm{DE}=0.25)$; con valores mayores a $30^{\circ} \mathrm{C}$ en las lluvias (Figura 3). Presentando la TSM una relación positiva con la abundancia del calamar dedal $\left(R^{2}=0.764\right)$.

De los 822 especímenes recolectados de calamar dedal, 746 fueron hembras y 76 machos, con intervalo de longitud de 21 a $110 \mathrm{~mm}$ LM en las hembras y de 21 a $93 \mathrm{~mm}$ LM en los machos (Figura 4A). Las tallas más impactadas por las redes de pesca se observan en las modas de entre 65 y $89 \mathrm{~mm}$ LM en hembras y de 40 y $80 \mathrm{~mm}$ LM en machos. Por otro lado, las tallas presentaron variación entre las zonas de muestreo, con diferencias significativas entre S92S94 ( $p=0.001)$ y S92-S95 ( $p=0.02)$ (Figura $2 C$ ).

El calamar dedal es un organismo dioico cuyo marcado dimorfismo sexual se observa en el brazo IV hectocotilizado en los machos, brazo modificado para la cópula; mientras que las hembras son más grandes y robustas, lo que se corrobora con las diferencias en talla y peso entre machos y hembras (K-S, $p<0.05)$. La relación entre $L M$ y el peso para hembras fue $p=$ $0.00013 L^{2.835}\left(R^{2}=0.97\right.$, Figura $\left.4 C\right)$ y en los machos $p=0.00015 L^{2} M^{2.775}\left(R^{2}=0.48\right.$; Figura 4D); con pendiente diferente de $3.0(p<0.05)$.

Por su parte, la proporción sexual tuvo una clara predominancia de hembras (Figura 4B), aunque en el mes de julio se registró el mayor número de machos $\left(7 \mathrm{H}: 1 \mathrm{M}, \chi^{2}=248.81, \mathrm{p}<0.05\right)$. Las hembras estuvieron presentes en todos los lances positivos para $L$. panamensis, con predominancia en profundidades de 10 a $30 \mathrm{~m}$; los machos por su parte, estuvieron presentes en $21.4 \%$ de las estaciones, pero con mayor abundancia entre 20 y $30 \mathrm{~m}$ de profundidad (Tabla 1, Figura 2C); con presencia de ejemplares de calamar dedal inmaduros de ambos sexos durante la temporada de secas, entre los 10 y $20 \mathrm{~m}$ de profundidad.

A partir del mes de mayo, las hembras y machos maduros se registraron juntos entre los 20 y 30 $\mathrm{m}$ de profundidad; mientras que en el mes de julio se presentó la mayor abundancia de ambos sexos, sobresaliendo la presencia de hembras maduras con parches de espermatangias en el manto como evi- 
Tabla 1. Abundancia de hembras y machos de Lolliguncula panamensis en el Golfo de Tehuantepec, por intervalo de profundidad y temporada climática.

\begin{tabular}{cccccccc}
\hline \multirow{3}{*}{ Temporada } & Sexo & \multicolumn{6}{c}{ Profundidad $(\mathrm{m})$} \\
\cline { 3 - 7 } & & $10-20$ & $21-30$ & $31-40$ & $41-50$ & $51-60$ & $61-70$ \\
Calamares/arrastre \\
\hline \multirow{2}{*}{ Lstiaje } & hembras & 32 & 3 & 8 & & 1 & \\
& machos & & & 1 & & \\
& hembras & 154 & 497 & 31 & 20 & & 1 \\
& machos & 5 & 5938 & 10 & & \\
\hline
\end{tabular}

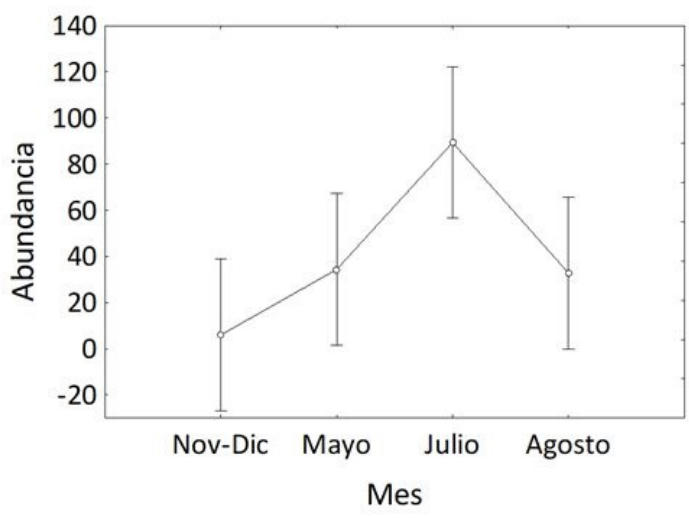

A
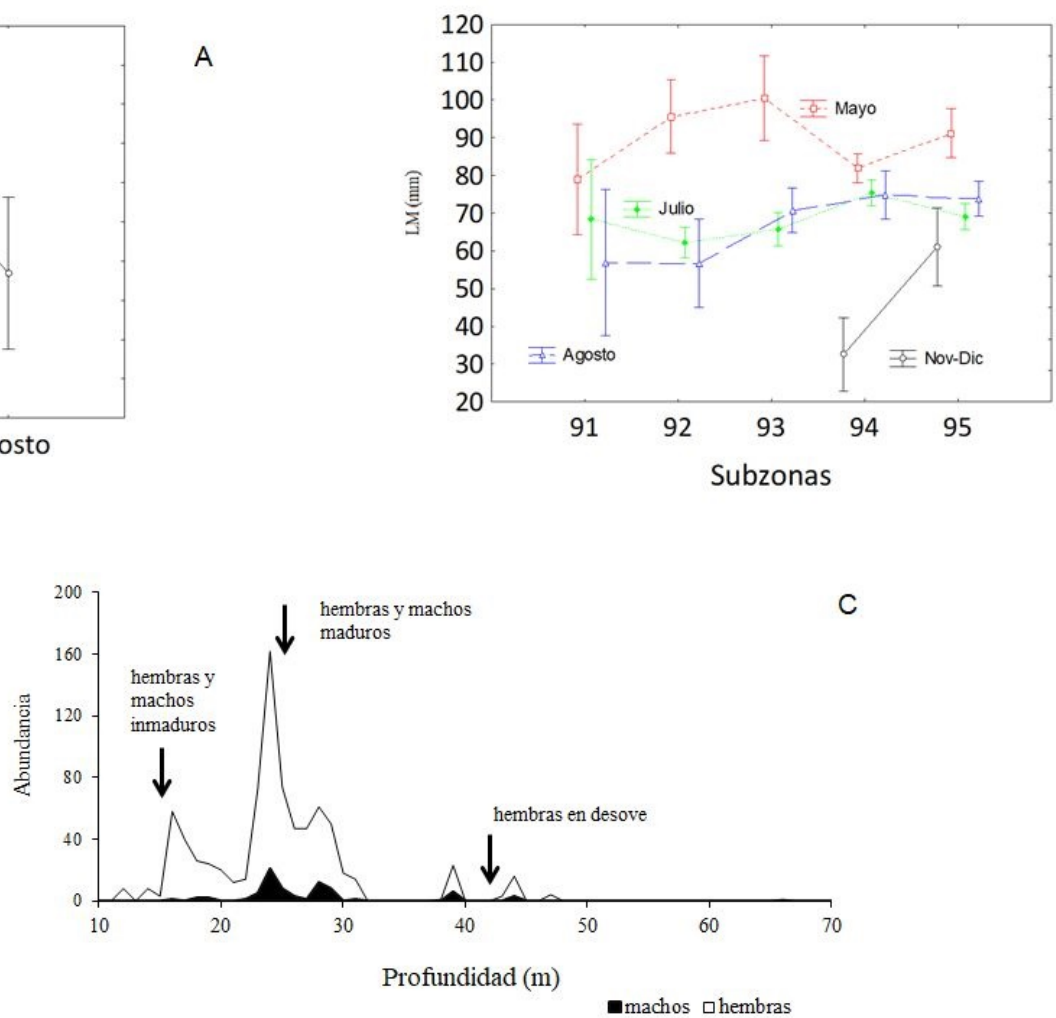

Figura 2. Lolliguncula panamensis. A) Comparación temporal de abundancias, $F(3,16)=5.0940, p=0.1154$; las barras verticales indican intervalos de confianza de 0.95. B) Comparaciones múltiples de talla entre sub-zonas de muestreo, $F(9,776)=6.0559, p<0.05$; las barras verticales indican intervalos de confianza de 0.95 . C) Distribución de la abundancia por intervalos de profundidad.

dencia de apareamiento. En agosto, entre los 30 y 40 $\mathrm{m}$ de profundidad se registraron hembras en desove con ovocitos hidratados en el ovario, además de un número muy reducido de machos (Figura $2 \mathrm{C}$ ).

\section{DISCUSIÓN}

El calamar dedal, forma parte de la fauna de acompañamiento de la pesquería de camarón en el Golfo de California y GT (Alejo-Plata et al. 2001,
Sánchez 2003, Arizmendi-Rodríguez et al. 2012b). En particular en el GT, la captura del calamar dedal es abundante en la temporada de veda del camarón en los meses de marzo a septiembre, como lo corroboran los resultados del presente estudio.

Con respecto a la distribución, el calamar dedal presenta diferentes respuestas a la variabilidad de la TSM, lo que sugiere un eventual beneficio derivado de un entorno oceánico cambiante. En este sentido, los resultados de la presente investigación indican que se presentó mayor abundancia de calamar dedal 

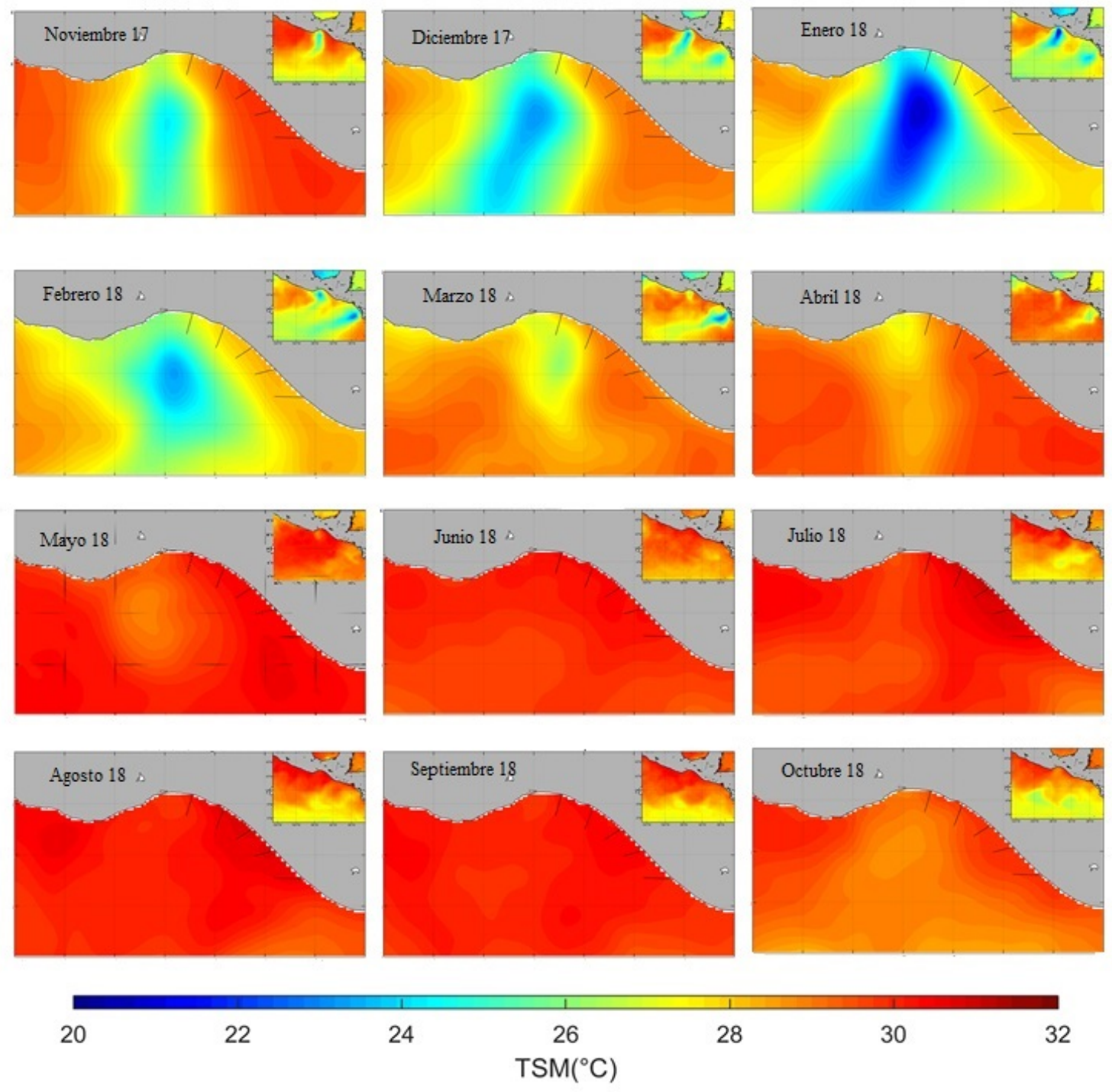

Figura 3. Temperatura superficial del mar (TSM) del Golfo de Tehuantepec en el periodo noviembre 2017 - octubre 2018.

en el Golfo de California en los meses fríos con TSM entre 17 y $22{ }^{\circ} \mathrm{C}$, que contrasta con la captura $50 \%$ menor en los meses cálidos (Arizmendi-Rodríguez et al. 2012b). Lo que difiere con las observaciones en la zona del GT, donde se tiene correlación positiva con la TSM. Situación que se puede deber a la plasticidad que presentan los calamares en su ciclo de vida en respuesta a diferencias ambientales (Forsythe 2004).

En el GT, la mayor presencia y abundancia de calamares con longitudes de más de $100 \mathrm{~mm}$ LM, se tuvo en la temporada de lluvia (julio-agosto; TSM $>30^{\circ} \mathrm{C}$ ), mientras que de noviembre a diciembre (TSM $\sim 26{ }^{\circ} \mathrm{C}$ ) se tuvo menor abundancia y calamares menores de $95 \mathrm{~mm}$ de LM. Al respecto, estudios previos, en el GT reportan baja abundancia (Tapia-García et al. 1994, Alejo-Plata et al. 2001, Sánchez 2003); lo que es contrastante con las altas abundancias encontradas en el presente estudio. Estas diferencias podrían explicarse con base a lo establecido por Doubleday et al. (2016), quienes reportan crecimiento en el tamaño poblacional de cefalópodos a nivel mundial en las últimas décadas. De modo 

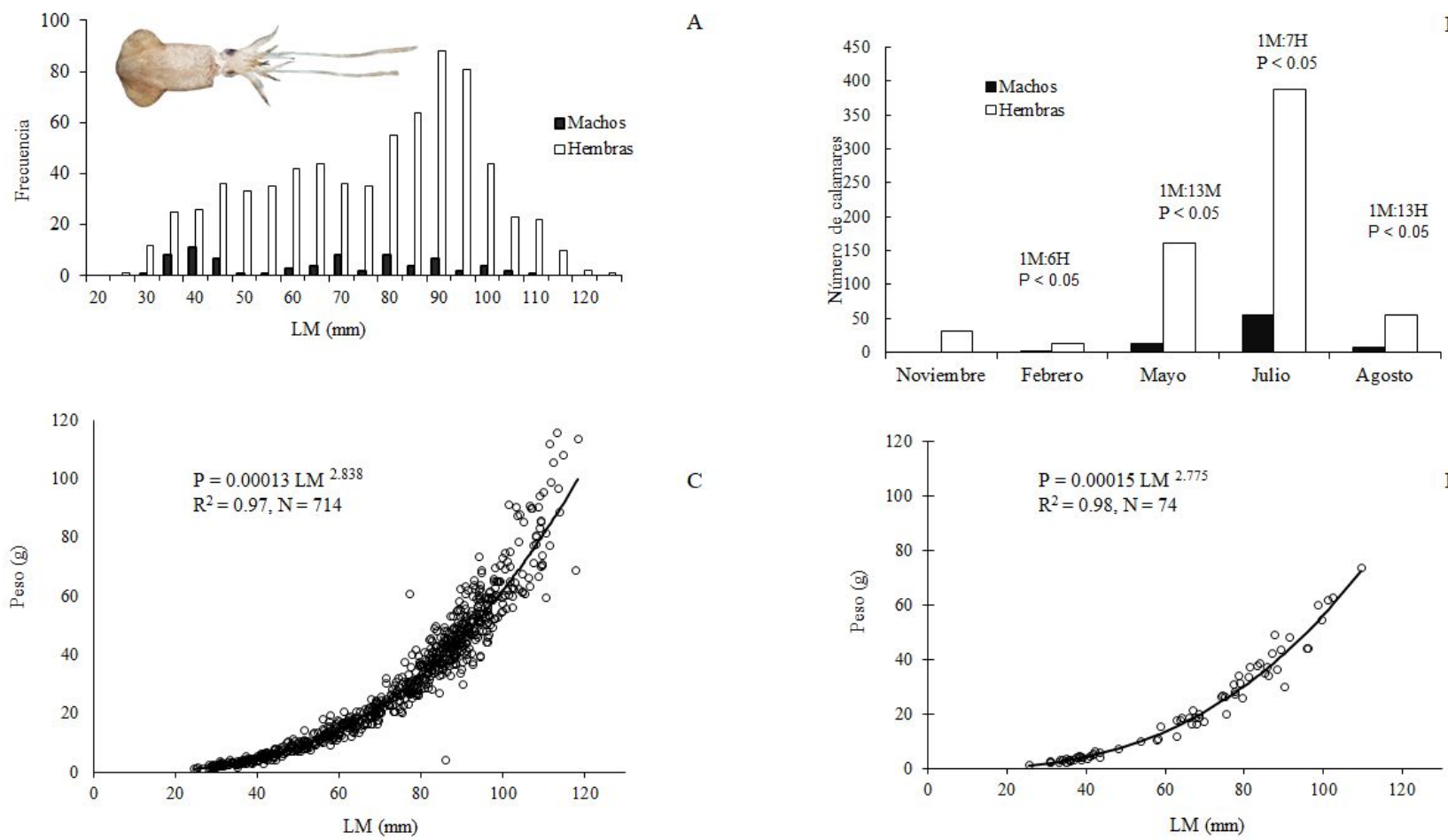

$\mathrm{C}$

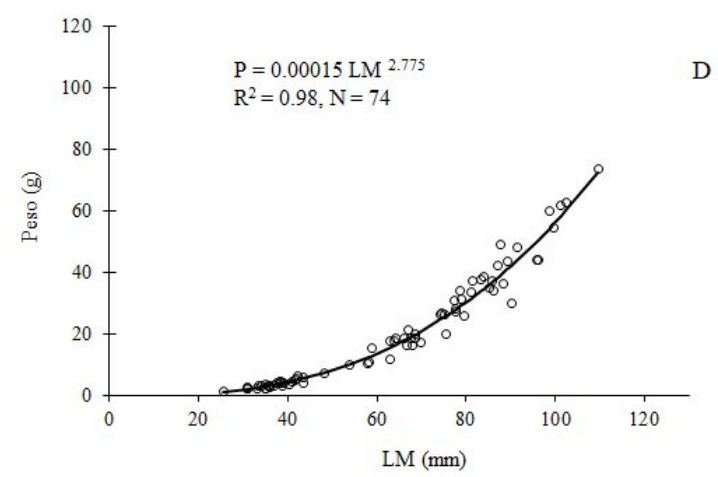

Figura 4. Lolliguncula panamensis. A. Distribución de frecuencias de la longitud dorsal del manto, hembras y machos; Relación talla-peso para hembras (B) y machos (C); D. Proporción sexual por mes de muestro.

particular, se ha observado un incremento cercano al $50 \%$ en la abundancia de calamares de la familia Loliginidae, debido a su condición como oportunistas ecológicos (Pelc y Jackson 2008). Así como por presentar un ciclo de vida corto y de rápido crecimiento, aunado a las diferentes estrategias reproductivas que los distinguen; además de la extrema flexibilidad y plasticidad de sus historias de vida. Estas características, permiten a estos calamares loliginidos adaptarse mucho más rápido a su entorno, que otras especies ante la dinámica de variables ambientales, como la temperatura (Pelc y Jackson 2008).

Por otra parte, existen estudios que indican diferencias en la distribución vertical entre las diferentes especies de Lolliguncula (ArizmendiRodríguez et al. 2012a, Arizmendi-Rodríguez et al. 2012b), sin embargo, los resultados obtenidos no indican la formación de agregaciones del calamar dedal que denoten actividades migratorias, como en otras especies de loliginidos reportadas por Sánchez
(2003) y Jereb et al. (2010). Asimismo, en los lances positivos para $L$. panamensis, no hubo registro de otras especies de Lolliguncula como L. diomedeae y $L$. argus, a pesar de que comparten la misma área de distribución (Jereb et al. 2010), lo que confirma diferencias en su distribución batimétrica, y puede ser resultado de una exclusión competitiva entre estas especies, como lo señaló Sánchez (2003) para $L$. diomedeae y L. panamensis.

Además, la mayor abundancia de L. panamensis observada entre 20 y $30 \mathrm{~m}$ de profundidad representó alrededor del $70 \%$ de organismos con los brazos cortados y en regeneración. Al respecto, León-Guzmán y Alejo-Plata (2019) mencionan que el calamar dedal exhibe una autotomía parcial y tiene la habilidad para regenerar sus brazos. Este comportamiento refuerza la idea de exclusión competitiva de esta especie. En lo que respecta al dimorfismo sexual por tallas, se sabe que los calamares loligínidos machos de muchas especies son de mayor talla que las 

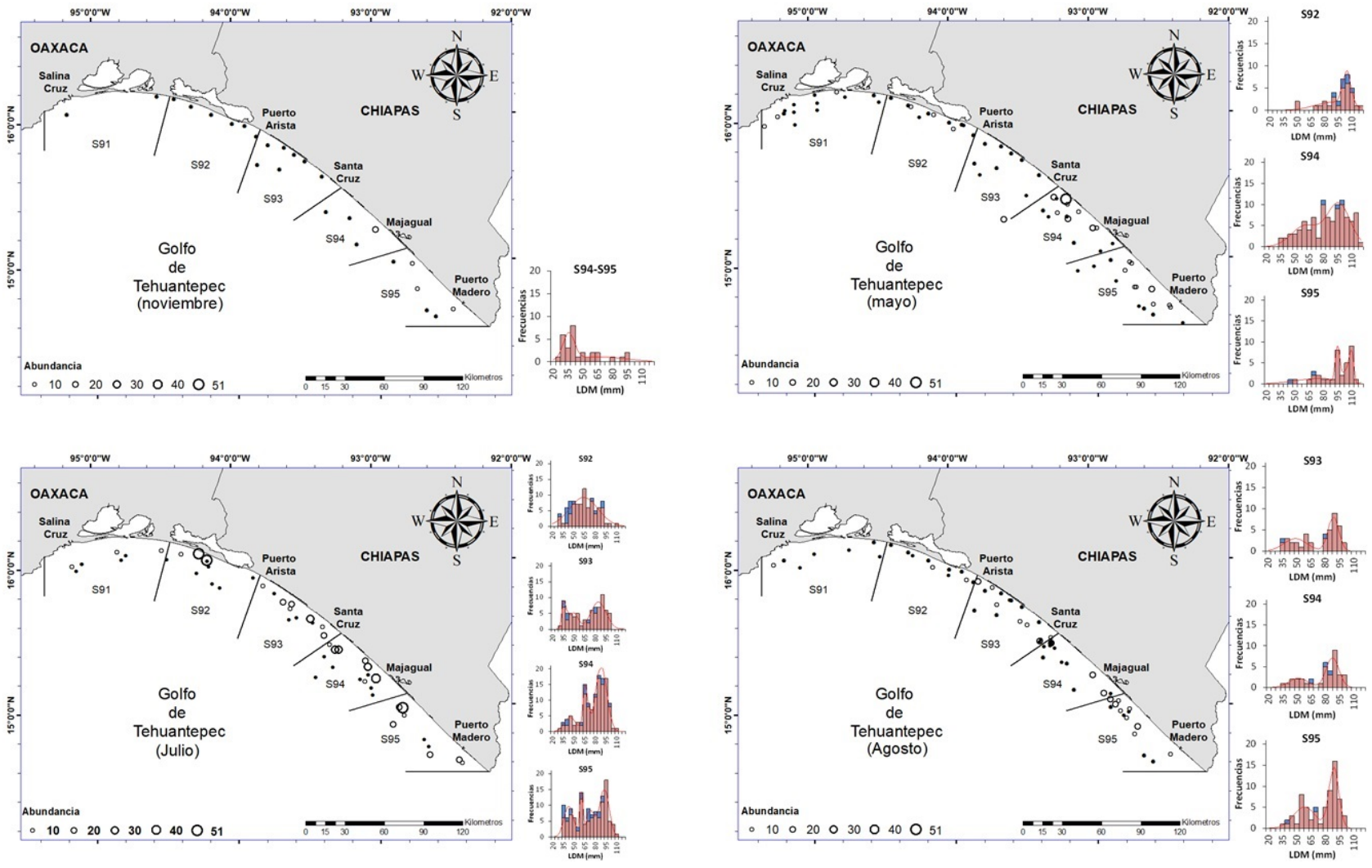

Figura 5. Distribución y abundancia espacio-temporal de L. panamensis en el Golfo de Tehuantepec. A) noviembre-diciembre, febrero; B) mayo; C) julio; D) agosto.

hembras (Perez et al. 2002, Rodrigues y Gasalla 2008). Pero contrario a lo observado por ArizmendiRodríguez et al. (2012a) quienes mencionan que las hembras de L. panamensis del golfo de California son de mayor talla y más abundantes en comparación con los machos; lo que coincide con lo observado para esta especie en el GT. Esta condición de dimorfismo sexual, también fue documentada en $L$. argus y $L$. diomedeae (Alejo-Plata et al. 2015, Alejo-Plata et al. 2016). Por lo que la presencia de machos pequeños y hembras de mayor talla parece ser un rasgo común en las poblaciones de Lolliguncula.

Por otro lado, la pendiente de la relación peso-longitud del manto, así como el peso por unidad de longitud de las hembras fue significativamente superior en comparación con los machos. Lo que se puede deber a que las hembras tienen una mayor inversión en los órganos reproductivos que en el crecimiento somático, lo que también se reporta para otros miembros de la familia Loliginidae (Sánchez 2003, Rodrigues y Gasalla 2008, Arizmendi-Rodríguez et al. 2012a).

La mayor abundancia de hembras maduras durante la temporada de lluvias, podría relacionarse con las diferencias en la proporción sexual que es mayor en las hembras y que puede ser consecuencia del comportamiento reproductivo de la especie. Por lo que la tendencia puede ser resultado de una captura selectiva de las hembras, más que por efecto de diferencia en la proporción de sexos. Mientras que en la temporada de secas, se registró una baja abundancia de hembras maduras, y presencia de hembras inmaduras y juveniles en los lances superficiales (10$20 \mathrm{~m}$ de profundidad). Al respecto, se conoce que los calamares en cada etapa de su ciclo de vida (paralarvas, juveniles, adultos) habitan diferentes áreas (Vidal 2014).

Además, las capturas en los diferentes niveles 
de la columna de agua, sugieren que en las lluvias $L$. panamensis migra hacía aguas poco profundas (10$20 \mathrm{~m}$ ) cercanas a la costa para aparearse y desovar. Por consiguiente, la mayor abundancia de hembras, asociada a la presencia de machos a partir de mayo, podría corresponder a un pulso de migración reproductiva. Al respecto, Arizmendi-Rodríguez et al. (2012a) reportan un comportamiento de agregación reproductiva de la especie en el Golfo de California. En julio se registró la mayor abundancia de machos maduros, con presencia de hembras maduras con parches de espermatangias en el manto; lo que evidencia posibles eventos de apareamiento. Durante agosto se observó la presencia de hembras con ovocitos hidratados en los ovarios, condición que indica un desove inminente, que puede extenderse a octubre. Al respecto, Arizmendi-Rodríguez et al. (2012a) mencionan que el calamar dedal se caracteriza por presentar desoves asincrónicos y múltiples, cuyos periodos se pueden extender durante varios meses.

\section{CONCLUSIONES}

Los resultados sugieren variación espaciotemporal de $L$. panamensis dentro del GT, en una estrecha relación con las temporadas de lluvias y secas; resultado quizá de la respuesta positiva de la especie al aumento de la TSM. Por consiguiente, las condiciones ambientales en la región podrían ser las adecuadas para la reproducción y crecimiento de $L$. panamensis.

\section{AGRADECIMIENTOS}

Al Centro Regional de Investigaciones Pesqueras de Salina Cruz del Instituto Nacional de la pesca y a su personal, por la oportunidad de participar en los viajes de pesca en 2017 y 2018. El financiamiento se obtuvo del proyecto Calamares: Recurso Pesquero alternativo para la costa de Oaxaca (CONACYT-PDCPN-1740). MCAP y AFGA agradecen a SNI-CONACYT.

\section{LITERATURA CITADA}

Alejo-Plata MC, Cerdenares-Ladrón de Guevara G. Herrera-Galñindo JE (2001) Cefalópodos lologínidos en la fauna de acompañamiento del camarón. Ciencia y Mar 5: 43-48.

Alejo-Plata MC, Gómez-Márquez JL, Herrera-Galindo JE (2015) Tallas, sexos y madurez gónadica del calamar dardo Lolliguncula diomedae (Cephalopoda: Loliginidae) en el Golfo de Tehuantepec, México. Research Journal of the Costa Rican Distance Education University 7: 79-87.

Alejo-Plata MC, Urbano-Alonso B, Ramírez-Castelán IA (2016) New records and biological data of Lolliguncula (Lolliguncula) argus Brakoniecki \& Roper, 1985 (Myopsida: Loliginidae) in the Gulf of Tehuantepec, Mexico. Latin American Journal of Aquatic Research 44: 855-859.

Alverson DL, Freeberg MH, Murawski SA, Pope JG (1994) A global assessment of fisheries bycatch and discards. FAO Fisheries Technical Paper. Rome. 339p.

Arizmendi-Rodríguez DI, Cruz-Escalona VH, Quiñones-Velázquez C, Salinas-Zavala CA (2011) Fedding habits of the Panama brief squid (Lolliguncula panamensis) in the Gulf of California, Mexico. Journal of Fisheries and Aquatic Sciences 6: 194-201.

Arizmendi-Rodríguez DI, Rodríguez-Jaramillo C, Quiñónez-Velázquez C, Salinas-Zavala CA (2012a) Reproductive indicators and gonad development of the Panama Brief squid Lolliguncula panamensis (Berry, 1911) in the Gulf of California, Mexico. Journal of Shellfish Research 31: 817-826.

Arizmendi-Rodríguez DI, Salinas-Zavala CA, Quiñones-Velázquez C, Mejía-Rebollo A (2012b) Abundance and distribution of the Panama brief squid, Lolliguncula panamensis (Teuthida: Liliginidae), in the Gulf of California. Ciencias Marinas 38: 31-45. 
Cardoso F, Hochberg FG (2013) Revision of the genus Lolliguncula Steentrup, 1881 (Cephalopoda: Loliginidae) off the Pacific coast of South America. Revista Peruana de Biología 20: 129-136.

DOF (2013) Norma Oficial Mexicana NOM-002-SAG/PESC-2013, Para ordenar el aprovechamiento de las especies de camarón en aguas de jurisdicción federal de los Estados Unidos Mexicanos. Diario Oficial de la Federación. 43p.

Doubleday ZA, Prowse TA, Arkhipkin A, Pierce GJ, Semmens J et al. (2016) Global proliferation of cephalopods. Current Biology 26: 387-407.

Forsythe JW. (2004) Accounting for the effect of temperature on squid growth in nature: from hypothesis to practice. Mar Freshwater Res 55: 331-339.

Gallegos-García A, Barberán-Falcon J (1998) Surgencia eólica. En: Tapia-García M (ed) El Golfo de Tehuantepec: El ecosistema y sus recursos. Universidad Autónoma Metropolitana-Iztapalapa. México. pp: 27-34.

Hastings NAJ, Peacock JB (1975) Statistical distributions in scientific work series: A handbook for students and practitioners. John Wiley \& Sons. New York. 130p.

INAPESCA (2012) Plan de manejo para la pesquería de camarón en el litoral del Océano Pacífico mexicano. Doc. Interno. SAGARPA. México. 144p.

Jereb P, Vecchione M, Roper CFE (2010) Family Loliginidae. In: Jereb P, Roper CFE (eds) Cephalopods of the world. An annotated and illustrated catalogue of species known to date, Vol. 2. FAO. Rome. pp: 38-117.

León Guzmán SS, Alejo-Plata MC (2019). Arms regeneration in the squid Lolliguncula panamensis (Mollusca: Cephalopoda). Latin American Journal of Aquatic Research 47(2): 356-360.

Lipinski MR, Underhill LG (1995) Sexual maduration insquid: quantum or continuum? African Journal of Marine Science 15: 207-223.

López-Martínez J, Herrera-Valdivia E, Rodríguez-Romero J, Hernández-Vázquez S (2010) Peces de la fauna de acompañamiento en la pesca industrial de camarón en el Golfo de California, México. Revista de Biología Tropical 58: 925-943.

Ortega-García S, Trigueros-Salmeron J, Rodríguez-Sanchéz R, Luch-Cota S, Villalobos H (2000) El golfo de Tehuantepec como un centro de actividad biológica y su importancia en las pesquerías. En: Lluch-Belda D, Elorduy-Garay J, Lluch-Cota S, Ponce-Díaz G (eds) BAC: Centros de actividad biológica del Pacífico mexicano. CIBNOR, CICIMAR, CONACYT. México. pp: 335-356.

Pecl GT, Jackson GD (2008) The potential impacts of climate change on inshore squid: biology, ecology and fisheries. Reviews in Fish Biology and Fisheries 18: 373-385.

Perez JAA, De Aguiar DC, Oliveira UC (2002) Biology and population dynamics of the long-finned squid Loligo plei (Cephalopoda: Loliginidae) in southern Brazilian waters. Fisheries Research 58: 267-279.

Reyes S, Mejía-Trejo A (1991) Tropical perturbations in the eastern Pacific and the precipitation field over northwestern Mexico in relation to ENSO phenomenon. International Journal of Climatology 11: 515-528.

Rodrigues AR, Gasalla MA (2008) Spatial and temporal patterns in size and maturation of Loligo plei and Loligo sanpaulensis (Cephalopoda: Loliginidae) in southeastern Brazilian waters, between $23 \mathrm{~S}$ and $27 \mathrm{~S}$. Scientia Marina 72: 631-643.

Romero-Centeno R, Zavala-Hidalgo J, Gallegos A, O'Brien JJ (2003) Tehuantepec isthmus wind climatology and ENSO signal. Journal of Climate 16: 2628-2639. 
Sánchez P (2003) Cephalopods from off the Pacific coast of Mexico: biologicalaspects of the most abundant species. Scientia Marina 67: 81-90.

Tapia-García M (1998) Evaluación ecológica de la ictiofauna demersal. En: Tapia-García M (ed) El Golfo de Tehuantepec: el ecosistema y sus recursos. Universidad Autónoma Metropolitana-Iztapalapa. México. pp: 103-128.

Tapia-García M, García-Abad MC, González-Medina G, Macuitl-Montes MC, Cerdenares-Ladrón de Guevara G (1994) Composición, distribución y abundancia de la comunidad de peces demersales del Golfo de Tehuantepec. Tropical Ecology 35: 229-252.

Vidal E (2014) Advances in Cephalopod Science: Biology, Ecology, Cultivation and Fisheries (Vol. 67). Academic Press. USA. 467p.

Zar HH (1999) Biostatistical analysis. Prentice Hall. New Jersey. 944p. 